\title{
Evaluation of Eustachian Tube Function in Mucosal Chronic Otitis Media: A Cost-Effective Method for Developing Countries
}

\author{
Vikram Kemmannu Bhat ${ }^{1}$ Gowri Sankar Marimuthu ${ }^{2}$ Kiran Herur ${ }^{1}$ Mona Yadav \\ ${ }^{1}$ Department of Otolaryngology, Head and Neck Surgery, Karnataka \\ Institute of Medical Sciences, Hubli, Karnataka, India \\ 2Department of Otolaryngology, Head and Neck Surgery, Jawaharlal \\ Institute of Post Graduate Medical Education and Research, \\ Puducherry, India

\begin{abstract}
Address for correspondence Vikram Kemmannu Bhat, MS, DNB, MNAMS, PhD, Department of Otolaryngology, Head and Neck Surgery, Karnataka Institute of Medical Sciences, Vidyanagar, Hubli 580021, Karnataka, India (e-mail: vikram.ent@gmail.com).
\end{abstract}

Ann Otol Neurotol ISO 2018;1:111-114

\begin{abstract}
Background In the developing nations, the sinonasal region is routinely screened in chronic otitis media, but Eustachian tube is not as it is not cost-effective. Hence, we innovated an inexpensive device named Eustachian barotubometer.

Materials and Methods A prospective case-control study was performed on mucosal type of chronic otitis media and traumatic tympanic perforation (100 cases each). Eustachian tube function was assessed by the Eustachian barotubometer (forced and physiological opening) and nasopharyngoscopy.

Results Eustachian tube dysfunction was found to be five times higher in cases than controls. Both forced opening function and physiological opening function were poorer in cases than controls. However, the difference was statistically significant only

Keywords

- chronic otitis media

- Eustachian tube function

- middle ear in physiological opening function $(p=0.003)$. Among the cases with dysfunctional tubes, $86.7 \%$ were hypofunctional, whereas $13.3 \%$ were totally blocked.

Conclusion Eustachian tube function was affected in $15 \%$ of cases of mucosal chronic otitis media as compared with controls in this study. This novel device could be conveniently used to help decide the right time to undertake middle ear surgery.
\end{abstract}

\section{Introduction}

Chronic otitis media (COM) is a common disease of the developing countries, and the surgical correction for the same continues to be a routine surgical procedure in the operation theater. The pathology of COM is multifactorial and mediated via the Eustachian tube (ET). However, ET function is hardly evaluated before undertaking any definitive middle ear surgery for various reasons. ET function evaluation, apart from being elaborate, is also inconsistent and unpredictable many times. ET evaluation in a perforated tympanic membrane (TM) requires tympanometer machines that are still not affordable in the developing countries, especially at the primary care level. In this background, we innovated an inexpensive device ( - Fig. 1) named as Eustachian barotubometer (EBT) that could evaluate ET function in a perforated TM. ${ }^{1}$
With this instrument, we tried to find out the need to evaluate the ET function in mucosal COM.

\section{Materials and Methods}

This study aimed to evaluate the ET function in mucosal COM (quiescent and inactive stages only) and compare with that of traumatic tympanic perforation (100 patients each). This was a prospective case-control study that was undertaken in a tertiary referral public hospital in a span of 1 year after clearance from the institutional ethics review board. All consecutive eligible adult patients with mucosal COM (cases) and traumatic tympanic perforation (controls) from the outpatient clinic were enrolled. Evaluation of physiologic and forced ET functions in mucosal COM and traumatic tympanic perforation was the main outcome. ${ }^{1}$ The assessment of 


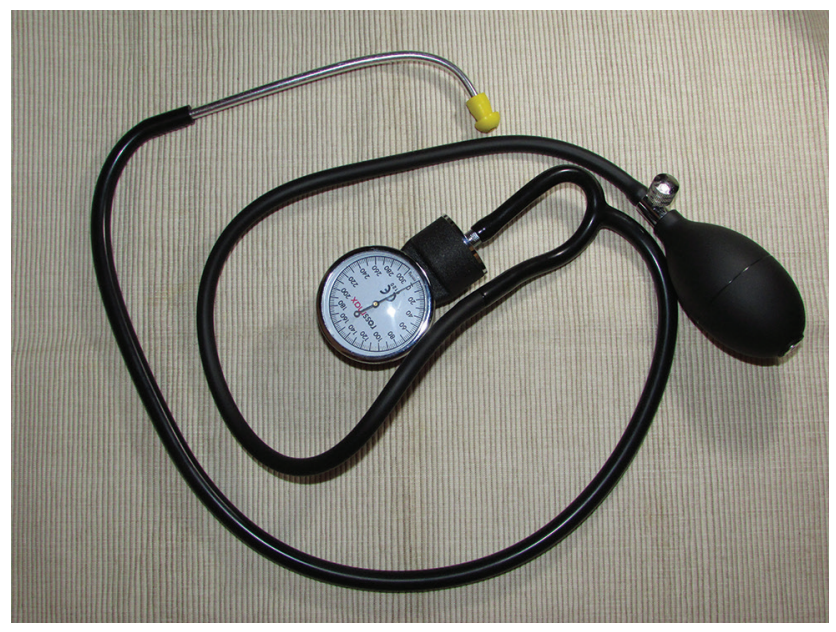

Fig. 1 Prototype of assembled Eustachian barotubometer.

pharyngeal end of ET by nasopharyngoscopy (NPS) was the secondary outcome.

Eustachian tube function was initially assessed by Valsalva's maneuver and NPS. In the former, the patient was asked to report whether he heard a hissing sound from the perforated ear. In the latter, he was asked to swallow during NPS and the pharyngeal opening of ET was evaluated. The ET function was further evaluated by the EBT. ${ }^{1}$

\section{Procedure of Eustachian Tube Function Evaluation}

For the forced opening test (FOT), the pressure was raised slowly in steps up to $50 \mathrm{~mm} \mathrm{Hg}$ for 2 minutes before declaring that the ET failed to force open. ${ }^{1}$ For physiological opening test (POT), the patient was asked to dry swallow four to five times without opening his mouth when the pressure in the ear canal was being raised slowly for 2 minutes (maximum pressure attained was $<30 \mathrm{~mm} \mathrm{Hg}$ ). The air pressure would then fall as soon as the ET opened. When an air leak was detected in the ear tip, it was replaced with another one and the test was repeated. Patients not achieving air seal on EBT were excluded from the study.

The presence or absence of the forced opening or physiologic opening of ET was noted.

\section{Observation and Results}

The patients' age ranged from 21 to 60 years (average age: 29.7 for cases and 35.4 for controls). Bilateral involvement of the ear was seen in $33 \%$ of cases. However, only one ear was considered in the study. Among the perforations, $6 \%$ were subtotal, $55 \%$ were large, $27 \%$ were moderate sized, and $12 \%$ small in size.

However, all controls had only unilateral involvement and had a small- to large-sized perforation. ET dysfunction was found to be five times higher in cases than controls. Both forced opening function and physiological opening functions were poorer in cases than controls (-Tables 1, 2). However, the difference was statistically significant only in physiological opening function. - Fig. 2 shows the results obtained from the EBT test in the cases and controls.

It was observed that apart from deviated nasal septum, none of the cases or controls in the study had any other pathology in the sinonasal region. The assessment findings of the pharyngeal end of ET by NPS in the cases and controls are given in $\boldsymbol{-}$ Table $\mathbf{3}$. It is to be noted that all the dysfunctional tubes were in the quiescent stage of COM. The tube function was normal in $85 \%$, hypofunctional in $13 \%$, and completely dysfunctional/blocked in $2 \%$.

\section{Discussion}

In the past, ET function was presumed to be normal, and it was thought that evaluation of the same was unnecessary before reconstructive middle ear surgery. ${ }^{2}$ However, now it is accepted that the disorder of the ET function is an important factor in the evolution of COM, restoration is crucial for successful surgical results. Unfortunately, till date, there is no foolproof method by which it can be evaluated accurately. Swarts et $\mathrm{al}^{3}$ have found a sensitivity of $74.2 \%$ and a specificity of $65.6 \%$ for identifying ET openings during a swallow by sonotubometry. Inflation deflation test was used by Kurien et al ${ }^{4}$ to assess ET and aditus patency. Sonotubometry was used by Virtanen et $\mathrm{al}^{5}$ to obtain a reliable picture of ET opening during swallowing and was found to be more physiological than negative-pressure equalization test. Munjal et $\mathrm{al}^{2}$ studied Valsalva's maneuver before and after tympanoplasty and found that it was a reliable qualitative test.

However, currently no single test could be considered a "gold standard" for the diagnosis of ET dysfunction, but there is some evidence that diagnostic accuracy can be improved by combining the results of different objective tests and patient-reported outcome measures. ${ }^{6}$ It has also been shown that the ET scoring system is a valid and reliable instrument in adult patients with chronic obstructive ET dysfunction. ${ }^{7}$ The panel on ET dysfunction agreed that this was a syndrome with a constellation of signs and symptoms suggestive of dysfunction and had three subtypes. ${ }^{8}$

Table 1 Results of physiological opening in the groups

\begin{tabular}{|l|l|l|l|}
\hline ET (POT) & Case & Control & Total \\
\hline ET patent & 85 & 97 & 182 \\
\hline ET not patent & 15 & 3 & 18 \\
\hline Total & 100 & 100 & 200 \\
\hline
\end{tabular}

Abbreviations: ET, Eustachian test; POT, physiologic opening test.

Chi-square $=8.79, p=0.003, p<0.01$. 
Table 2 Results of forced opening in the groups

\begin{tabular}{|l|l|l|l|}
\hline EBT (FOT) & Case & Control Total \\
\hline ET patent & 96 & 99 & 195 \\
\hline ET not patent & 4 & 1 & 5 \\
\hline Total & 100 & 100 & 200 \\
\hline
\end{tabular}

Abbreviations: EBT, Eustachian barotubometer; ET, Eustachian test; FOT, forced opening test.

Chi-square $=1.85, p=0.174, p>0.05$.

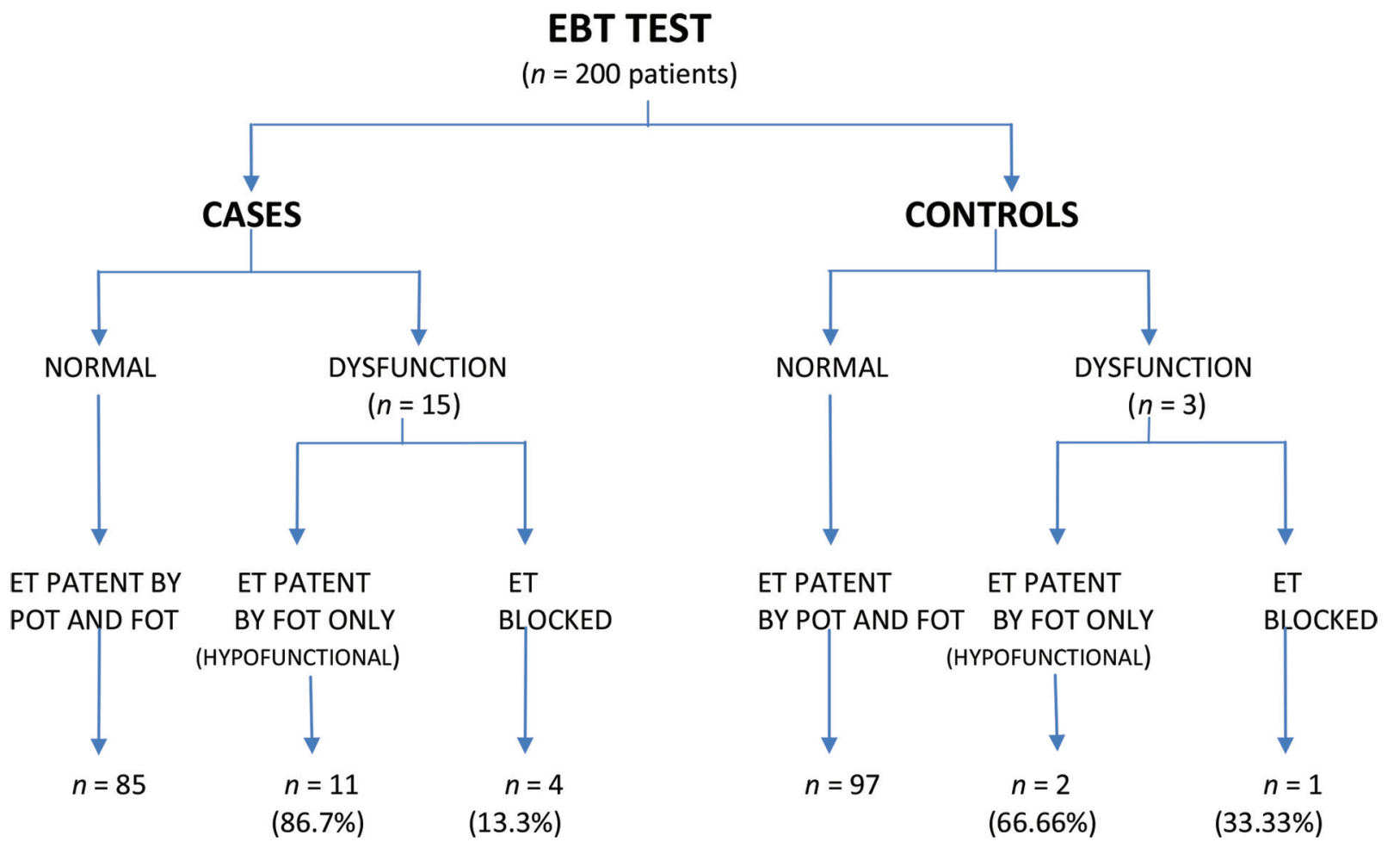

Fig. 2 Flowchart of study design and recruitment of patients. EBT, Eustachian barotubometer; ET, Eustachian test; FOT, forced opening test; POT, physiologic opening test.

Table 3 Assessment of Eustachian tube opening by nasopharyngoscopy in the groups

\begin{tabular}{|l|l|l|l|}
\hline NPS & Case & Control Total \\
\hline ET patent & 88 & 98 & 186 \\
\hline ET not patent & 12 & 2 & 14 \\
\hline Total & 100 & 100 & 200 \\
\hline
\end{tabular}

Abbreviations: ET, Eustachian test; NPS, nasopharyngoscopy.

Chi-square $=7.68, p=0.006, p<0.01$.

In our previous study, the EBT was compared and validated against a standard tympanometer, and the interpretations of the various observations are explained in a flowchart. ${ }^{1} \mathrm{We}$ have found that this instrument is an inexpensive alternative to the tympanometer. Also, this instrument is robust, rugged, portable, and does not require power supply; hence, it is suitable even for the primary health care centers. In this study, only cases in the quiescent and inactive stage of mucosal COM were included, and hence $15 \%$ of them were found to have reduced ET function. The figure would have probably been higher in the active stage. Cases from pediatric age group were excluded from this study as the chance of finding enlarged adenoids and other upper respiratory tract infections was high. Moreover, the problem of air leaks through ear tips was found to be greater in children. The ET function was evaluated by Valsalva's maneuver, and forced opening parameter of EBT aimed at finding a mechanical or anatomical factor impairing tube function. This was further supported by the evidence on NPS, showing no significant mechanical cause of ET dysfunction. 
Many times, it is difficult to distinguish a physiological structure such as an anatomical variation from a definitive pathological focus in the nose and paranasal sinuses, as revealed in our previous study. ${ }^{9}$ Also, it is difficult to establish to what extent the disease focus directly or indirectly affects the functioning of the ET. It has been shown that there is a tendency for poor ET function in patients with nasal septal deviation. ${ }^{10}$ As revealed in our previous study, even though a majority (>90\%) of the cases with COM have some or the other "predisposing disease focus" in the sinonasal region, it may not necessarily lead to an ET dysfunction. ${ }^{9}$ Our data show that the ET function could get affected even in patients with traumatic TM perforation. This means that the ET dysfunction may not necessarily lead to COM all the times.

Whether the outcomes of middle ear surgery in all these ET affected cases would be different needs to be studied further. Prasad et $\mathrm{al}^{11}$ agree that ET function assessment should be a part of the preoperative workup for middle ear surgery. Chauhan and Chauhan ${ }^{12}$ stated that diagnostic nasal endoscopy or videonasopharyngoscopy was a useful adjuvant along with ET function tests for qualitative assessment of the nasopharynx.

Tubal mucosal edema seemed to be a significant intrinsic factor affecting physiological function of ET in this study. Sadé and Luntz ${ }^{13}$ found that the ET lumen was more affected in the cartilaginous part of the ET and the inflammatory reaction was more in the bony part, when normal and pathological temporal bones were compared.

ET function is unpredictable, and uncertainty looms over the recovery of the function in the dysfunctional cases. Even the episodes and duration of dysfunction are not predictable all the times. This makes the assessment of ET function and its impact on middle ear even the more complicated. However, at least at the time of tympanoplasty, a functioning ET is required so that it would be successful. ${ }^{14}$ Our device can precisely do that and can hence help decide the right time for surgery. The therapeutic use of this instrument to restore ET functions has been tried in children, and encouraging results have been found. Further studies are required in this field to ascertain the actual benefits of this therapy.

\section{Conclusion}

ET function is affected significantly in COM cases as compared with controls. The altered function of ET in COM is predominantly hypofunctional and mainly in the quiescent stage in this study. Hence it appears reasonable to evaluate the ET function routinely in COM before undertaking any surgery in the middle ear. This novel device can be conveniently used to help decide the right time to undertake the ear surgery, especially at the peripheral health care level.

\section{Note}

This paper was presented as a poster by the first author at the 16th British Academic Conference in Otolaryngology held at Manchester, United Kingdom, from July 4 to 6, 2018.

\section{References}

1 Bhat VK, Kumar PR, Nag M, Hegde J. Comparison of a Eustachian barotubometer with a tympanometer to evaluate Eustachian tube function in chronic suppurative otitis media. J Otolaryngol Head Neck Surg 2009;38(4):456-461

2 Munjal M, Chopra H, Gupta SN. Valsalva and Politzer tests in myringoplasty. Indian J Otol 2000;6(1):15-18

3 Swarts JD, Teixeira MS, Banks J, El-Wagaa J, Doyle WJ. A method to assess the accuracy of sonotubometry for detecting Eustachian tube openings. Eur Arch Otorhinolaryngol 2015;272(9):2111-2119

4 Kurien R, Chrisolyte S, Rupa V. Inflation-deflation test as a predictor of aditus patency in patients with chronic suppurative otitis media. Indian J Otolaryngol Head Neck Surg 2009;61(3):169-172

5 Virtanen H, Palva T, Jauhiainen T. Comparative preoperative evaluation of Eustachian tube function in pathological ears. Ann Otol Rhinol Laryngol 1980;89(4 Pt 1):366-369

6 Smith ME, Tysome JR. Tests of Eustachian tube function: a review. Clin Otolaryngol 2015;40(4):300-311

7 Schröder S, Lehmann M, Sauzet O, Ebmeyer J, Sudhoff H. A novel diagnostic tool for chronic obstructive Eustachian tube dysfunction-the Eustachian tube score. Laryngoscope 2015;125(3):703-708

8 Schilder AGM, Bhutta MF, Butler CC, et al. Eustachian tube dysfunction: consensus statement on definition, types, clinical presentation and diagnosis. Clin Otolaryngol 2015;40(5):407-411

9 Bhat VK, Paramu AT, Nagachar S. Predisposing sinonasal disease foci: do they really affect mucosal chronic otitis media? Annals of Otology and Neurotology 2018 [In Press]

10 Akyıldız MY, Özmen OA, Demir UL, et al. Should nasal function be considered prior to tympanoplasty? J Int Adv Otol 2018;14(1):53-57

11 Prasad KC, Hegde MC, Prasad SC, Meyappan H. Assessment of Eustachian tube function in tympanoplasty. Otolaryngol Head Neck Surg 2009;140(6):889-893

12 Chauhan B, Chauhan K. A comparative study of Eustachian tube functions in normal and diseased ears with tympanometry and videonasopharyngoscopy. Indian J Otolaryngol Head Neck Surg 2013;65(Suppl 3):468-476

13 Sadé J, Luntz M. Eustachian tube lumen: comparison between normal and inflamed specimens. Ann Otol Rhinol Laryngol 1989;98(8 Pt 1):630-634

14 Sheehy JL. Testing Eustachian tube function. Ann Otol Rhinol Laryngol 1981;90(6 Pt 1):562-565 